\title{
Towards a Pattern Language for Aspect-Based Design
}

\author{
Maarten Bynens $\quad$ Wouter Joosen \\ DistriNet, Dept. of Computer Science, K.U.Leuven \\ Celestijnenlaan 200A \\ B-3001 Leuven, Belgium \\ \{maarten.bynens,wouter.joosen\}@cs.kuleuven.be
}

\begin{abstract}
With the maturation and widespread adoption of AOSD comes the need for patterns and best practices to solve recurring problems. Such patterns have been documented in different publications. Besides a conclusive catalog, a system to relate the patterns and drive the process of pattern selection would bear significant benefits. In this position paper, we designate the road towards a pattern language for aspect-based design and describe the initial steps.
\end{abstract}

\section{Categories and Subject Descriptors}

D.2.11 [Software Engineering]: Software Architecturepatterns; D.2.2 [Software Engineering]: Design Tools and Techniques-object-oriented design methods

; D.3.3 [Programming Languages]: Language Constructs and Features-patterns

\section{General Terms}

Design, Documentation

\section{Keywords}

Design patterns, pattern language, AOP, AOSD

\section{INTRODUCTION}

A pattern is a solution to a problem in a particular context. To find the right pattern in a given situation is often harder than expected. At this moment non-experienced aspect developers will need to search through different sources of pattern descriptions as no decisive catalogue of AO design patterns exists.

A pattern language is the ideal instrument to drive the process of pattern selection. There is still a large gap between what is currently available and having a genuine pattern language. In this position paper, we designate the road towards a pattern language for aspect-based design and describe the initial steps.

Permission to make digital or hard copies of all or part of this work for personal or classroom use is granted without fee provided that copies are not made or distributed for profit or commercial advantage and that copies bear this notice and the full citation on the first page. To copy otherwise, to republish, to post on servers or to redistribute to lists, requires prior specific permission and/or a fee.

PLATE'09, March 3, 2009, Charlottesville, Virginia, USA.

Copyright 2009 ACM 978-1-60558-453-9/09/03 ...\$5.00.

\section{PATTERN LANGUAGE}

A pattern language [1] is a coherent whole of interrelated patterns. It describes the relationships between its constituent patterns and as a result identifies different pattern categories and layers to differentiate between patterns used in different contexts and patterns of different scale respectively. The result is a graph with as root the layer of architectural patterns, which addresses our large-scale problem, broadly outlines the solution provided by the language and defines a selection process by traversing the graph following paths and variation points. These paths and variation points in the language are defined by the different types of relationships present in the language (e.g. pattern that uses other patterns, patterns solving a similar problem, conflicting patterns, ... )

\section{FIRST STEPS}

We base our approach on the work by Noble [10]. Patterns are divided in 3 fragments, separating architectural patterns, design patterns and programming idioms. These fragments serve as the layers of the pattern language, where each layer uses patterns from lower-level layers. In each fragment the relationships between the patterns are described in more detail using 3 basic relationship types:

- Uses: part-of, composes multiple patterns into composite pattern

- Refines: specialization, addresses a more specific problem (e.g. Factory Method refines Template Method)

- Conflicts: provide mutually exclusive solutions to a similar problem

\subsection{Collecting design patterns}

A number of AO design patterns have been described in the past, but there is still a lack, as proven by this workshop. The most familiar patterns are (as described in $[4,5,6,7$, $3,9,8,2])$ : template pointcut, template advice, pointcut method, marker interface, pointcut interface, naming and annotation conventions, container introduction, chained advice, participant, annotation introduction, wormhole, director, border control, worker object creation, ...

\subsection{Differentiating categories and fragments}

We see that most of these patterns deal with the usability, reusability and evolution of the aspect. We can divide them in three categories: 
1. Deal with the fragile binding between aspects and base program:

- abstract pointcut

- marker interface: abstract from types in the aspect and connect these types to the base program separately

- container introduction: introduce members based on a marker interface

- participant: connect an abstract pointcut for each subsystem separately and within that subsystem

- annotation introduction: write aspects in terms of annotations and add these to the base program separately

- director: abstract aspect with multiple roles as nested interfaces

- border control: set of pointcuts that delimit certain regions in the base application

2. Aim to achieve a more flexible design of the aspect itself:

- template advice: decompose advice into hook methods describing more variable parts

- template pointcut: decompose pointcut into hook pointcuts, describing more variable parts

- pointcut method: encapsulate dynamic condition in method to be called from advice

- chained advice: specify an order between advice from different aspects running at the same join point

3. Make base program more aspect-aware by making join points more explicit:

- pointcut interface: provide an interface with stable pointcuts supported by the base code

- naming and annotation conventions

4. Solving a domain-specific problem:

- worker object creation: creating objects that handle asynchronous method executions

5. Solving a problem of programming with objects:

- wormhole: transport context information throughout a method call chain without the need for parameters

We can classify pointcut method, container introduction, wormhole, border control and worker object creation as programming idioms, while the others fit in the design fragment (although there are no strict criteria for either fragment).

A candidate for the architectural fragment is Reusable aspect, which makes use of patterns from the categories described above and aims to provide aspects that are reusable in different contexts with a flexible binding to the base code that is easy to configure.

\subsection{Identifying pattern relationships}

Within each category (but also between different categories), we can identify relationships between the patterns. Without going into too much detail we give a few examples:

- marker interface and annotation introduction refine an abstract pattern Indirect Connection

- template pointcut uses abstract pointcut as a mandatory part of its specification

- participant is in conflict with chained advice (chained advice splits an aspect based on functionality running on overlapping join points, while participant splits an aspect based on the different subsystems with relevant join points, as a result there will be no overlapping join points and chained advice is not applicable)

A natural evolution of identifying the relationships is the emergence of abstract (or virtual) and helper patterns. Such patterns take the same role as abstract classes and utility classes in software development. They provide common mechanisms or common problem descriptions used by other patterns. E.g. the abstract pattern Indirect Connection mentioned above describes the technique of an aspect using abstractions to define its pointcuts, advice, etc. and a separate connection of these abstractions with a concrete base application.

\subsection{Describing selection process}

The selection process is tightly coupled to the relationships in the language. Uses relations drive the developer from one pattern to another, while refines and conflicts make up variation points. It is very likely that the described relationships will not entirely suffice to specify all the possible constraints that are relevant in selecting the appropriate set of patterns for a specific situation.

\section{CONCLUSION}

It should be clear that still a lot of work needs to be done, to reach the goal of a full pattern language. These initial steps need to be augmented with further listing of patterns (of all categories and granularity) and experience of using them to identify relationships.

\section{REFERENCES}

[1] C. Alexander. A Pattern Language. 1977.

[2] M. Bynens, B. Lagaisse, W. Joosen, and E. Truyen. The elementary pointcut pattern. In BPAOSD '0\%: Proceedings of the 2nd workshop on Best practices in applying aspect-oriented software development, page 2, New York, NY, USA, 2007. ACM.

[3] W. G. Griswold, K. Sullivan, Y. Song, M. Shonle, N. Tewari, Y. Cai, and H. Rajan. Modular software design with crosscutting interfaces. IEEE Softw., 23(1):51-60, 2006.

[4] S. Hanenberg and A. Schmidmeier. Idioms for building software frameworks in aspectj. AOSD Workshop on Aspects, Components, and Patterns for Infrastructure Software, 2003.

[5] S. Hanenberg and R. Unland. Using and Reusing Aspects in AspectJ. Workshop on Advanced Separation of Concerns, OOPSLA, 2001. 
[6] S. Hanenberg, R. Unland, and A. Schmidmeier. AspectJ Idioms for Aspect-Oriented Software Construction. In Proceedings of EuroPLoP, pages 617-644, 2003.

[7] R. Laddad. AspectJ in Action: Practical Aspect-Oriented Programming. Manning Publications Co., Greenwich, CT, USA, 2003.
[8] B. Lagaisse and W. Joosen. Decomposition into elementary pointcuts: A design principle for improved aspect reusability. In SPLAT, 2006.

[9] R. Miles. AspectJ Cookbook. O'Reilly Media, Inc., 2004.

[10] J. Noble. Towards a pattern language for object oriented design. In In: Technology of Object Oriented Langauges 28, pages 2-13, 1998. 